\title{
20. TESTING A HIGHER RESOLUTION INTERSTITIAL-WATER METHOD FOR THE OCEAN DRILLING PROGRAM1
}

\author{
Ray E. Cranston ${ }^{2}$
}

\begin{abstract}
Ocean Drilling Program inorganic geochemistry procedures routinely overlook more than $99 \%$ of the sediment column. Present and past biogeochemical reactions alter the sediment record; however, most of these reaction zones are bypassed by the normal methods where samples are collected every $30 \mathrm{~m}$. A new approach to increase resolution was introduced during Leg 119. Ten milliliters of sediment provided interstitial-water samples for ammonia, silica, sulfate, magnesium, and calcium analyses. The new method introduced some systematic differences in concentrations, as well as some decrease in precision. A number of advantages, however, may warrant using the method in some instances. In cases where routine interstitial-water data showed anomalous results, core sections were retrieved from the storage facility and resampled. The new high-resolution procedure was used to provide water samples in cases were water contents were low and routine squeezing could not recover pore water.
\end{abstract}

\section{INTRODUCTION}

A number of potential problems and missed opportunities can result from Ocean Drilling Program (ODP) procedures used to collect interstitial-water samples. More than $99 \%$ of the sedimentary column is not studied. Routine ODP interstitial-water samples are $300-\mathrm{mL}$ whole-round sediment samples collected at 30 -m intervals. When water content drops below 20\% (wet weight), there is a tendency not to continue squeezing, as the pore-water yield is low.

Many dissolved components are in equilibrium states controlled by temperature, redox conditions, and ionic strength. ODP samples are squeezed at room temperature rather than at in-situ temperature. Removing pore water at different temperatures affects ionic concentrations, whereas processing at $4^{\circ} \mathrm{C}$ tends to minimize the effect (DeLange et al., 1989). Normal ODP handling operations allow pore waters to be exposed to atmospheric oxygen, which can alter the composition of chemical species in the water (Loder et al., 1978). The use of fresh (drill) water during coring operations (as well as periodically during splitting operations) can alter the ionic strength of pore-water samples. Simple changes in shipboard procedures can minimize the oxidation effects and provide samples that can be analyzed for redox-sensitive species (e.g., Fe, $\mathrm{U}$, and $\mathrm{S}^{2-}$ ), which is problematic when using the routine procedure.

A higher resolution interstitial-water sampling program was introduced during Leg 119. The major goal was to collect porewater samples every few decimeters to meters, rather than every few tens of meters. Pore-water samples could be collected from very dry sediments in cases where routine squeezing was not effective. The sediment sample size was reduced to $10 \mathrm{~mL}$, rather than the $300-\mathrm{mL}$ full-round sample required for the routine method. Samples were stored and processed under cold, inert atmospheric conditions to minimize temperature and oxidation effects. The new method uses a dilution step to recover pore water, which has an impact on equilibrium conditions. Because some dilution from drill water can occur during routine proce-

\footnotetext{
${ }^{1}$ Barron, J., Larsen, B., et al., 1991. Proc. ODP, Sci. Results, 119: College Station, TX (Ocean Drilling Program).

2 Geological Survey of Canada, Bedford Institute of Oceanography, Box 1006, Dartmouth, Nova Scotia, Canada, B2Y 4A2.
}

dures, it was felt that dilution effects were of interest to the program and therefore warranted further study.

In cases where regular geochemical results suggested chemical reactions were occurring, core sections were resampled in the cold-storage room at 0.3 - to 0.5 -m intervals to identify the exact location and characteristics of the reaction (Chambers and Cranston, this volume). Core description is not always a useful indicator of in-situ reactions. In many cases, pore-water profiles varied a great deal even though the sediment column appeared to be uniform. Taking one geochemistry sample every $30 \mathrm{~m}$ in what appears to be a uniform sedimentary regime can be a serious oversimplification.

\section{METHODS AND RESULTS}

Samples were collected at $0.3-$ to $3-\mathrm{m}$ intervals for selected sites. Approximately $10 \mathrm{~mL}$ of wet sediment was sealed in a plastic sample bag and refrigerated. Within $12 \mathrm{hr}$ of splitting the core, each sample was transferred to a $50-\mathrm{mL}$ centrifuge tube in a helium-flushed glove bag, capped, and refrigerated.

Within $24 \mathrm{hr}$ of splitting, $5 \mathrm{~mL}$ of degassed deionized water (i.e., oxygen-free) was added to each sample in the He-filled glove bag. The sample was vigorously mixed using a Vortex mixer and allowed to stand for $1 \mathrm{hr}$ at $4^{\circ} \mathrm{C}$. The cold, capped tubes were centrifuged at $5000 \mathrm{rpm}$ for $5 \mathrm{~min}$ using an IEC HNSII centrifuge. Three milliliters of the pore-water-deionized-water mixture was filtered through a $0.45-\mu \mathrm{m}$ membrane filter. Shipboard analyses for dissolved silica, chlorinity, sulfate, and ammonia were done using routine ODP methods (Barron, Larsen, et al., 1989). Shipboard analyses of $\mathrm{Mg}$ and $\mathrm{Ca}$ in the higher resolution samples were not possible because of time constraints. Diluted pore-water samples were stored in tightly capped scintillation vials and returned to my laboratory for analyses of magnesium and calcium using flame atomic absorption spectroscopy methods.

Comparative data for the regular interstitial-water results and the new dilution method are contained in Table 1. Figures 1 through 5 show the results for the comparative methods for ammonia, magnesium, calcium, silica, and sulfate, respectively. Included with each figure is the 1:1 equal-concentration line, the number of cases plotted, and the correlation coefficient. Ammonia and sulfate analyses could not be done on all high-resolution samples because of time constraints; thus, there were only 51 comparable cases for the ammonia and sulfate data 
Table 1. Comparison of pore-water results from the new and regular methods of analyses.

\begin{tabular}{|c|c|c|c|c|c|c|c|c|c|c|}
\hline \multirow{2}{*}{$\begin{array}{l}\text { Depth } \\
\text { (mbsf) }\end{array}$} & \multicolumn{2}{|c|}{ Silica $(\mu \mathrm{M})$} & \multicolumn{2}{|c|}{ Sulfate $(\mathrm{mM})$} & \multicolumn{2}{|c|}{$\begin{array}{c}\text { Ammonia } \\
(\mathrm{mM})\end{array}$} & \multicolumn{2}{|c|}{$\begin{array}{l}\text { Magnesium } \\
(\mathrm{mM})\end{array}$} & \multicolumn{2}{|c|}{ Calcium (mM } \\
\hline & New & Regular & New & Regular & New & Regular & New & Regular & New & Regula \\
\hline \multicolumn{11}{|l|}{ Site 736} \\
\hline 4.25 & 831 & 725 & - & 17.5 & - & 0.14 & 55.0 & 51.6 & 12.2 & 10.1 \\
\hline 21.25 & 967 & 767 & - & 24.8 & - & 0.28 & 48.7 & 51.7 & 10.5 & 10.2 \\
\hline 42.07 & 1120 & 922 & - & 24.5 & - & 0.25 & 55.8 & 54.0 & 12.2 & 10.5 \\
\hline 57.25 & 907 & 836 & - & 25.7 & - & 0.25 & 49.3 & 53.1 & 10.3 & 10.4 \\
\hline 83.75 & 1010 & 862 & - & 23.0 & - & 0.31 & 54.4 & 54.6 & 11.6 & 10.6 \\
\hline 115.23 & 1140 & 984 & - & 25.4 & 0.41 & 0.33 & 54.6 & 54.0 & 11.3 & 10.1 \\
\hline 153.65 & 1220 & 999 & 23.4 & 16.0 & 0.38 & 0.38 & 56.5 & 53.5 & 11.7 & 10.0 \\
\hline 176.25 & 1120 & 990 & - & 24.8 & - & 0.41 & 54.1 & 52.3 & 12.4 & 9.9 \\
\hline 207.98 & 1160 & 1001 & - & 25.4 & - & 0.39 & 49.7 & 52.5 & 11.0 & 9.9 \\
\hline 233.90 & 1260 & 1130 & - & 25.7 & - & 0.39 & 49.0 & 53.4 & 10.4 & 9.8 \\
\hline 265.95 & 1650 & 1428 & - & 24.8 & - & 0.39 & 49.3 & 53.4 & 10.7 & 10.2 \\
\hline 297.60 & 2170 & 1939 & - & 24.5 & 0.38 & 0.40 & 49.9 & 52.4 & 9.5 & 10.2 \\
\hline 325.29 & 2140 & 1897 & - & 24.8 & - & 0.38 & 47.4 & 52.0 & 7.9 & 10.1 \\
\hline 365.55 & 1930 & 1943 & - & 25.1 & - & 0.39 & 47.1 & 52.2 & 9.7 & 9.6 \\
\hline
\end{tabular}

Site 737

$\begin{array}{rrrrlllllll}2.10 & 684 & 576 & 25.1 & 27.4 & - & 0.07 & 48.9 & 52.0 & 12.9 & 11.5 \\ 21.10 & 827 & 717 & 22.5 & 27.1 & 0.07 & 0.11 & 47.9 & 49.8 & 19.5 & 17.1 \\ 59.10 & 1070 & 856 & 15.2 & 24.1 & 0.10 & 0.17 & 40.4 & 44.1 & 27.0 & 26.6 \\ 86.10 & 1460 & 1012 & 16.3 & 23.0 & - & 0.22 & 35.8 & 38.8 & 32.5 & 32.4 \\ 116.10 & 1160 & 939 & 15.6 & 21.5 & 0.25 & 0.26 & 35.3 & 35.8 & 33.9 & 38.8 \\ 144.60 & 1390 & 1240 & 12.1 & 20.7 & 0.30 & 0.30 & 26.4 & 32.4 & 34.5 & 44.8 \\ 373.01 & 1190 & 1099 & 15.4 & 11.8 & 0.11 & 0.25 & 1.3 & 2.0 & 78.8 & 04.8 \\ 404.57 & 493 & 293 & 16.6 & 16.6 & 0.34 & 0.25 & 0.7 & 1.5 & 85.6 & 27.5\end{array}$

Site 738

$\begin{array}{rrccccccccc}2.10 & 806 & 683 & 28.0 & 25.8 & 0.01 & 0.02 & 47.5 & 53.5 & 12.0 & 11.1 \\ 17.12 & 802 & 607 & 28.0 & 25.5 & 0.01 & 0.01 & 50.1 & 53.8 & 12.6 & 11.4 \\ 55.08 & 532 & 517 & - & 22.9 & - & 0.02 & 49.2 & 53.2 & 14.0 & 12.8 \\ 82.12 & 533 & 549 & - & 23.7 & - & 0.02 & 52.6 & 51.5 & 16.7 & 13.9 \\ 106.10 & 633 & 541 & 28.0 & 23.7 & 0.01 & 0.01 & 52.9 & 50.3 & 18.8 & 14.9 \\ 139.30 & 657 & 669 & - & 24.0 & - & 0.01 & 48.4 & 48.5 & 19.2 & 16.5 \\ 168.30 & 585 & 677 & - & 24.0 & - & 0.01 & 48.8 & 47.6 & 21.0 & 18.0 \\ 206.70 & 634 & 558 & - & 23.4 & - & 0.01 & 48.5 & 47.1 & 23.5 & 19.5 \\ 218.00 & 552 & 592 & - & 23.7 & - & 0.01 & 46.3 & 46.7 & 24.7 & 20.1 \\ 245.42 & 749 & 547 & - & 23.4 & - & 0.01 & 42.8 & 45.2 & 23.9 & 21.8 \\ 276.20 & 550 & 498 & - & 22.9 & - & 0.02 & 38.7 & 43.2 & 26.9 & 23.8 \\ 333.70 & 740 & 532 & - & 22.9 & - & 0.02 & 36.2 & 41.0 & 30.9 & 28.0 \\ 401.30 & 695 & 465 & - & 23.7 & - & 0.02 & 35.8 & 38.5 & 31.8 & 30.7\end{array}$

Site 739

$\begin{array}{rrrrrrrrrrr}26.02 & 843 & 136 & 26.0 & 26.7 & 0.60 & 0.14 & 12.0 & 42.6 & 7.2 & 19.5 \\ 132.40 & 700 & 140 & 17.5 & 17.7 & 0.92 & 0.46 & 5.8 & 40.6 & 4.7 & 17.9 \\ 146.58 & 1300 & 482 & 16.3 & 13.6 & 1.54 & 0.69 & 10.7 & 37.7 & 6.5 & 15.6 \\ 161.40 & 586 & 237 & 16.0 & 17.4 & 1.48 & 0.69 & 8.3 & 42.4 & 5.3 & 14.3 \\ 174.24 & 826 & 489 & 13.4 & 11.9 & 1.29 & 0.90 & 20.5 & 38.8 & 8.5 & 14.6 \\ 203.10 & 1130 & 412 & 13.3 & 10.8 & 1.52 & 1.05 & 19.8 & 36.6 & 8.0 & 13.9 \\ 233.50 & 889 & 592 & 10.1 & 9.4 & 1.40 & 1.03 & 26.0 & 37.3 & 10.2 & 14.0 \\ 262.50 & 809 & 615 & 11.1 & 8.4 & 1.30 & 1.09 & 26.3 & 36.0 & 10.0 & 14.1 \\ 301.00 & 508 & 485 & 14.5 & 8.4 & 1.20 & 1.08 & 21.6 & 35.0 & 8.2 & 13.9 \\ 328.11 & 159 & 98 & 11.7 & 10.8 & 1.40 & 0.91 & 12.5 & 35.8 & 5.8 & 13.2 \\ 358.66 & 118 & 94 & 10.9 & 10.5 & 1.50 & 1.03 & 12.4 & 33.1 & 5.9 & 13.8 \\ 397.70 & 121 & 106 & 11.1 & 8.1 & 1.20 & 0.94 & 16.8 & 33.1 & 7.1 & 13.4 \\ 415.50 & 200 & 175 & 11.6 & 8.1 & 1.00 & 0.76 & 21.2 & 31.8 & 8.4 & 13.8 \\ 445.58 & 340 & 98 & 11.8 & 6.0 & 1.70 & 1.01 & 3.1 & 28.3 & 4.0 & 13.8 \\ 465.43 & 133 & 79 & 13.7 & 6.0 & 1.50 & 1.01 & 12.7 & 26.2 & 7.3 & 14.4\end{array}$

Site 744

$\begin{array}{rrrllllllll}0.90 & 878 & 714 & 28.0 & 28.7 & 0.01 & 0.00 & 53.7 & 52.5 & 12.1 & 11.0 \\ 16.10 & 716 & 596 & 28.0 & 28.7 & 0.01 & 0.01 & 53.2 & 52.7 & 12.9 & 11.0 \\ 25.59 & 676 & 605 & 28.0 & 29.1 & 0.01 & 0.00 & 50.7 & 52.5 & 12.6 & 11.7 \\ 44.64 & 751 & 605 & 28.0 & 27.6 & 0.01 & 0.01 & 52.8 & 51.6 & 13.5 & 12.4 \\ 82.57 & 843 & 640 & 28.0 & 27.3 & 0.01 & 0.00 & 49.3 & 50.0 & 14.8 & 14.2 \\ 111.10 & 801 & 656 & 28.0 & 28.4 & 0.01 & 0.00 & 49.3 & 49.1 & 15.8 & 15.4 \\ 139.60 & 1034 & 723 & 28.0 & 25.5 & 0.01 & 0.00 & 46.7 & 48.7 & 17.5 & 16.7 \\ 159.30 & 698 & 620 & 28.0 & 28.4 & 0.01 & 0.00 & 47.4 & 47.9 & 18.0 & 17.3 \\ 11.69 & 840 & 667 & 28.0 & 28.5 & 0.01 & 0.00 & 53.2 & 52.7 & 12.9 & 11.2 \\ 21.19 & 1048 & 612 & 28.0 & 27.3 & 0.01 & 0.00 & 50.7 & 52.7 & 12.7 & 11.5\end{array}$


Table 1 (continued).

\begin{tabular}{|c|c|c|c|c|c|c|c|c|c|c|}
\hline \multirow{2}{*}{$\begin{array}{l}\text { Depth } \\
\text { (mbsf) }\end{array}$} & \multicolumn{2}{|c|}{ Silica $(\mu \mathrm{M})$} & \multicolumn{2}{|c|}{ Sulfate (mM) } & \multicolumn{2}{|c|}{$\begin{array}{c}\text { Ammonia } \\
(\mathrm{mM})\end{array}$} & \multicolumn{2}{|c|}{$\begin{array}{l}\text { Magnesium } \\
(\mathrm{mM})\end{array}$} & \multicolumn{2}{|c|}{ Calcium (mM) } \\
\hline & New & Regular & New & Regular & New & Regular & New & Regular & New & Regular \\
\hline \multicolumn{11}{|l|}{ Site 745} \\
\hline 2.70 & 801 & 689 & 28.3 & 29.4 & 0.05 & 0.10 & 69.0 & 52.7 & 12.9 & 11.2 \\
\hline 2.20 & 776 & 735 & 28.6 & 29.4 & 0.05 & 0.03 & 68.4 & 52.3 & 12.8 & 10.4 \\
\hline 16.70 & 921 & 728 & 24.9 & 28.4 & 0.17 & 0.18 & 59.3 & 51.9 & 11.1 & 11.9 \\
\hline 45.20 & 772 & 794 & 25.3 & 25.9 & 0.38 & 0.31 & 53.9 & 48.0 & 13.1 & 13.5 \\
\hline 73.70 & 780 & 761 & 24.7 & 24.8 & 0.44 & 0.36 & 68.5 & 46.0 & 18.0 & 15.1 \\
\hline 102.20 & 834 & 913 & 22.6 & 23.2 & 0.49 & 0.40 & 52.0 & 42.6 & 15.6 & 16.0 \\
\hline 130.70 & 972 & 996 & 22.7 & 22.0 & 0.52 & 0.36 & 46.7 & 40.7 & 16.3 & 16.7 \\
\hline 141.20 & 1136 & 946 & 22.6 & 21.6 & 0.54 & 0.39 & 49.6 & 39.9 & 17.3 & 17.5 \\
\hline 160.20 & 1069 & 1005 & 21.4 & 21.2 & 0.50 & 0.44 & 46.1 & 38.2 & 17.7 & 18.0 \\
\hline 188.70 & 1168 & 1010 & 22.0 & 19.4 & 0.59 & 0.46 & 41.2 & 36.6 & 18.0 & 18.8 \\
\hline \multicolumn{11}{|l|}{ Site 746} \\
\hline 176.50 & 969 & 948 & 18.3 & 21.2 & 0.47 & 0.50 & 43.0 & 37.3 & 19.2 & 19.9 \\
\hline 195.50 & 957 & 1017 & 16.1 & 19.8 & 0.48 & 0.38 & 40.2 & 35.2 & 19.0 & 20.4 \\
\hline 220.00 & 1056 & 944 & 16.9 & 17.4 & 0.75 & 0.43 & 38.4 & 34.0 & 19.3 & 21.5 \\
\hline 244.40 & 1367 & 1054 & 17.6 & 17.2 & 0.72 & 0.45 & 33.6 & 32.6 & 18.0 & 21.6 \\
\hline
\end{tabular}

Note: - indicates that analyses were not done.

whereas there were 74 comparable cases for the silicate, magnesium, and calcium data.

Each sample was weighed before the interstitial water was recovered and after freeze-drying to determine the amount of pore water and sediment in the original sample. A dilution factor (Table 2), calculated from the chlorinity data, was applied to correct for the $5 \mathrm{~mL}$ of deionized water that was added to each sample.

Platinum and calomel reference electrodes, standardized in Zobell solution, were used to measure the redox potential of the sediment samples (Whitfield, 1969). Potential voltages were acquired on a strip chart recorder for $90 \mathrm{~s}$ to account for electrode drift. Redox potential was calculated from the potential difference relative to the standard hydrogen electrode. Precision was estimated to be $\pm 0.2 \mathrm{pE}$ units.

Geochemical analyses of freeze-dried sediment and pore-water samples were carried out at my land-based laboratory. Methods and results are reported elsewhere (Cranston, this volume). Solid-phases analyses for $\mathrm{pE}$, opaline-leachable $\mathrm{Si}$, total $\mathrm{Al}$, water content (wet-weight basis), mean particle size, organic carbon, inorganic carbon, and dilution factor are included in Table 2 for the 74 samples where both pore-water methods were used. Table 3 contains correlation coefficients between calculated concentration differences (e.g., Si difference $=\mathrm{Si}$ concentration for regular method - Si concentration for new method) and the associated geochemical parameters in Table 2. This was done to determine which geochemical conditions had an influence on the analytical results.

\section{COMPARING THE METHODS}

The correlations between the two methods for each of the five chemical species (Figs. 1 through 5 ) are highly significant ( $p$ $<.001$ ), suggesting that the two methods are comparable in a relative sense. Each 8 - to 10 -mL sample of wet sediment contained 3 to $4 \mathrm{~mL}$ of pore water that was diluted with $5 \mathrm{~mL}$ of deionized water. The average dilution for the 74 samples considered in Table 1 was $1: 2.5$. It is well known that dilution of aqueous salt solutions in contact with soil or sediments tends to increase the concentration of divalent cations on the solid phase in exchange for the monovalent ions released to solution (Sayles and Mangelsdorf, 1977; Bohn et al., 1985). In the present study, ammonia release should occur due to the dilution step, while
$\mathrm{Mg}$ and $\mathrm{Ca}$ should be lost from solution. Sodium and potassium analyses were not done aboard ship for the regular interstitial-water samples, and, thus, comparative data for diluted and undiluted samples are not available.

\section{Ammonia}

Comparative results show that ammonia concentrations (Fig. 1) tended to be higher using the new dilution method for concentrations above $0.5 \mathrm{mM}$, suggesting that ammonia was released when the ionic strength of the pore water was reduced, as was predicted in the preceding discussion. The difference in ammonia concentrations between the two methods (ammonia determined by the regular method - ammonia determined by the new method) was most strongly related, in an opposite manner, with the dilution factor (Table $3, r=-0.74$ ). As the dilution factor increased, the difference between the methods became more negative (i.e., the results for the new dilution method were higher). The largest difference between the methods occurred for the Site 739 samples (Table 1), where water contents were lower and organic carbon concentrations were higher, compared to the other sites (Table 2). The results in Table 3 show that the concentration difference between the methods is strongly related to water content $(r=0.69)$ and to organic carbon $(r=-0.70)$. When cases with dilution factors of less than 2.5 are selected, the dilution method concentrations are an average of $8 \%$ higher than the regular method results (standard deviation of the mean $\pm 6 \%, n=28$, calculated from Table 1).

\section{Magnesium}

Magnesium concentrations (Fig. 2) were noticeably lower for the new method for some samples, suggesting that $\mathrm{Mg}$ uptake occurred during dilution, as was predicted previously. The largest deviations occurred for samples from Site 739 (Table 1), which had exceptionally low water contents $(15 \% \pm 3 \%, n=$ 15 , compared to $49 \% \pm 15 \%, n=59$, for other sites) as well as high dilution factors $(4.8 \pm 2.3, n=15$, compared to $1.9 \pm$ $0.4, n=59$, for other sites). This suggests that the dilution method is useful for $\mathrm{Mg}$ only if the dilution factor is kept below $1: 2.5$. The correlation analyses in Table 3 show that the observed differences in $\mathrm{Mg}$ concentrations relate most strongly to the dilution factor, and to a lesser extent to water content. When cases with dilution factors less than 2.5 are selected, the dilution 
Table 2. Geochemical data for samples on which both interstitial-water methods were used.

\begin{tabular}{rrrrrrrrr}
\hline $\begin{array}{c}\text { Depth } \\
\text { (mbsf) }\end{array}$ & $\mathrm{pE}$ & $\begin{array}{c}\text { Opaline } \\
\text { silica } \\
(\%)\end{array}$ & $\begin{array}{c}\text { Total } \\
\text { aluminium } \\
(\%)\end{array}$ & $\begin{array}{c}\text { Water } \\
\text { content } \\
(\% \text { wet })\end{array}$ & $\begin{array}{c}\text { Mean } \\
\text { size } \\
(\mu \mathrm{m})\end{array}$ & $\begin{array}{c}\text { Organic } \\
\text { carbon } \\
(\%)\end{array}$ & $\begin{array}{c}\text { Inorganic } \\
\text { carbon } \\
(\%)\end{array}$ & $\begin{array}{c}\text { Dilution } \\
\text { factor }\end{array}$ \\
\hline Site 736 & & & & & & & & \\
4.25 & 5.1 & 9.60 & 2.75 & 45.00 & 5.8 & 0.39 & 0.03 & 1.73 \\
21.25 & 2.9 & 6.20 & 4.56 & 46.10 & 6.4 & 0.64 & 0.08 & 1.81 \\
42.07 & 3.4 & 10.70 & 1.58 & 63.00 & 6.4 & 0.46 & 0.14 & 1.87 \\
57.25 & 3.7 & 2.40 & 5.66 & 34.00 & 13.7 & 0.29 & 0.09 & 1.83 \\
83.75 & 3.7 & 12.60 & 0.83 & 41.90 & 4.7 & 0.50 & 0.11 & 1.77 \\
115.23 & 3.6 & 11.20 & 1.26 & 33.50 & 8.9 & 0.43 & 0.06 & 1.85 \\
153.65 & 4.6 & 12.90 & 0.40 & 36.50 & 5.8 & 0.48 & 0.10 & 2.11 \\
176.25 & 5.0 & 14.90 & 0.61 & 44.00 & 5.3 & 0.47 & 0.11 & 1.73 \\
207.98 & 4.4 & 8.30 & 0.87 & 31.10 & 5.5 & 0.43 & 0.21 & 1.76 \\
233.90 & 3.9 & 9.50 & 0.99 & 44.90 & 6.3 & 0.38 & 0.06 & 2.07 \\
265.95 & 4.3 & 13.00 & 0.97 & 62.70 & 5.5 & 0.32 & 0.85 & 1.87 \\
297.60 & 3.6 & 22.20 & 0.37 & 59.20 & 4.9 & 0.26 & 0.11 & 1.99 \\
325.29 & 3.9 & 16.00 & 0.47 & 59.10 & 4.5 & 0.29 & 0.91 & 2.14 \\
365.55 & 3.9 & 13.90 & 0.38 & 60.40 & 5.0 & 0.26 & 0.53 & 1.92
\end{tabular}

Site 737

$\begin{array}{rrr}2.10 & 4.6 & 16.40 \\ 21.10 & 4.6 & 15.30 \\ 59.10 & 4.8 & 12.80 \\ 86.10 & 4.4 & 10.90 \\ 116.10 & 5.1 & 12.70 \\ 144.60 & 5.1 & 9.80 \\ 373.01 & 4.4 & 2.80 \\ 404.57 & 3.2 & 1.40\end{array}$

$\begin{array}{ll}1.92 & 72.80 \\ 1.13 & 73.60 \\ 1.82 & 65.80 \\ 2.83 & 62.30 \\ 0.93 & 64.30 \\ 1.27 & 70.20 \\ 6.01 & 25.70 \\ 4.77 & 28.10\end{array}$

$\begin{array}{rr}4.0 & 0.48 \\ 5.5 & 0.45 \\ 4.8 & 0.40 \\ 6.9 & 0.34 \\ 6.1 & 0.48 \\ 6.8 & 0.58 \\ 7.7 & 0.08 \\ 13.2 & 0.06\end{array}$

$\begin{array}{ll}0.08 & 1.59 \\ 0.12 & 1.56 \\ 0.02 & 2.01 \\ 0.04 & 1.94 \\ 0.03 & 1.55 \\ 0.01 & 1.83 \\ 0.22 & 2.05 \\ 1.02 & 2.21\end{array}$

Site 738

$\begin{array}{rll}2.10 & 8.0 & 4.40 \\ 17.12 & 8.2 & 2.50 \\ 55.08 & 6.3 & 0.10 \\ 82.12 & 6.5 & 0.50 \\ 106.10 & 6.8 & 0.70 \\ 139.30 & 6.1 & 0.70 \\ 168.30 & 6.0 & 0.30 \\ 206.70 & 5.8 & 0.40 \\ 218.00 & 6.5 & 0.60 \\ 245.42 & 6.3 & 0.50 \\ 276.20 & 4.8 & 0.50 \\ 333.70 & 4.8 & 1.30 \\ 401.30 & 5.6 & 1.20\end{array}$

0.95
1.29
0.18
0.03
0.18
0.53
0.08
0.86
0.32
0.01
0.59
0.49
1.18

48.40

40.10

38.10

37.70

37.60

36.40

33.20

30.50

33.70

30.30

22.90

24.20

\section{$8.6 \quad 0.04$}

$\begin{array}{llll}8.6 & 0.04 & 7.36 & 1.63\end{array}$

$\begin{array}{llll}4.9 & 0.04 & 7.13 & 2.28 \\ 3.7 & 0.04 & 0.58 & 1.88\end{array}$

$\begin{array}{llll}3.6 & 0.05 & 0.76 & 2.26\end{array}$

$\begin{array}{llll}3.6 & 0.05 & 0.76 & 2.26 \\ 3.8 & 0.03 & 0.45 & 2.28\end{array}$

$\begin{array}{llll}3.8 & 0.03 & 0.45 & 2.28 \\ 3.4 & 0.03 & 0.70 & 2.52\end{array}$

$\begin{array}{llll}3.2 & 0.03 & 0.77 & 2.10\end{array}$

$\begin{array}{llll}3.2 & 0.07 & 0.89 & 2.54\end{array}$

$\begin{array}{llll}3.2 & 0.07 & 0.89 & 2.54 \\ 3.3 & 0.05 & 0.37 & 2.14\end{array}$

$\begin{array}{llll}3.1 & 0.05 & 0.64 & 2.33\end{array}$

$\begin{array}{llll}3.5 & 0.06 & 0.05 & 2.91\end{array}$

Site 739

$\begin{array}{rr}26.02 & 2.9 \\ 132.40 & 3.6 \\ 146.58 & 2.9 \\ 161.40 & 3.9 \\ 174.24 & 4.3 \\ 203.10 & 4.1 \\ 233.50 & 3.7 \\ 262.50 & 4.3 \\ 301.00 & 3.4 \\ 328.11 & 3.9 \\ 358.66 & 4.4 \\ 397.70 & 3.9 \\ 415.50 & 3.7 \\ 445.58 & 3.1 \\ 465.43 & 3.6\end{array}$

$$
\begin{aligned}
& 0.30 \\
& 0.40 \\
& 1.30 \\
& 0.10 \\
& 0.90 \\
& 0.30 \\
& 1.30 \\
& 1.50 \\
& 0.30 \\
& 0.60 \\
& 0.40 \\
& 0.70 \\
& 0.90 \\
& 0.60 \\
& 0.70
\end{aligned}
$$

\subsection{6}

$$
\begin{aligned}
& 5.41 \\
& 5.19 \\
& 4.99
\end{aligned}
$$$$
3.74
$$

2.72

4.16

5.38

3.77

4.14

4.78

3.23

4.22

4.49

6.74

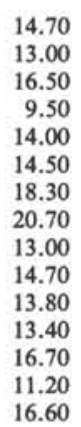

$\begin{array}{ll}3.4 & 0.32 \\ 4.3 & 1.26 \\ 4.6 & 1.07 \\ 6.0 & 1.42 \\ 4.7 & 0.58 \\ 4.1 & 0.43 \\ 4.3 & 0.52 \\ 5.4 & 0.62 \\ 4.2 & 0.49 \\ 5.3 & 0.57 \\ 4.0 & 0.58 \\ 4.9 & 0.57 \\ 4.7 & 0.58 \\ 4.9 & 0.33 \\ 5.9 & 0.68\end{array}$

$\begin{array}{rr}0.04 & 6.29 \\ 0.01 & 11.47 \\ 0.10 & 5.27 \\ 0.14 & 5.63 \\ 0.07 & 3.34 \\ 0.26 & 4.15 \\ 0.15 & 2.80 \\ 0.30 & 2.64 \\ 0.12 & 3.16 \\ 0.12 & 4.50 \\ 0.06 & 4.18 \\ 0.01 & 3.27 \\ 0.10 & 4.37 \\ 0.03 & 7.41 \\ 0.06 & 3.13\end{array}$

Site 744

$\begin{array}{rrr}0.90 & 7.8 & 13.20 \\ 16.10 & 8.2 & 10.30 \\ 25.59 & 8.5 & 4.30 \\ 44.64 & 7.3 & 1.20 \\ 82.57 & 7.3 & 1.80 \\ 111.10 & 7.6 & 0.50 \\ 139.60 & 6.8 & 2.60 \\ 159.30 & 6.7 & 0.30 \\ 11.69 & 8.2 & 9.00 \\ 21.19 & 8.3 & 2.90\end{array}$

$$
\begin{aligned}
& 0.79 \\
& 1.86 \\
& 0.75 \\
& 0.32 \\
& 0.83 \\
& 0.87 \\
& 0.71 \\
& 0.80 \\
& 0.75 \\
& 0.97
\end{aligned}
$$

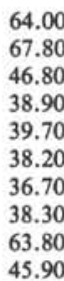

5.4
4.5
3.0
3.3
3.3
4.2
3.8
3.4
4.8
3.2

0.11
0.08
0.04
0.03
0.04
0.03
0.04
0.04
0.06

$\begin{array}{ll}3.78 & 1.71 \\ 0.21 & 1.77 \\ 6.94 & 1.68 \\ 8.29 & 1.79 \\ 7.80 & 1.76 \\ 8.34 & 1.72 \\ 7.79 & 1.96 \\ 8.50 & 1.79 \\ 2.66 & 1.64 \\ 7.28 & 1.77\end{array}$


Table 2 (continued).

\begin{tabular}{ccccccccc}
\hline $\begin{array}{c}\text { Depth } \\
(\mathrm{mbsf})\end{array}$ & $\mathrm{pE}$ & $\begin{array}{c}\text { Opaline } \\
\text { silica } \\
(\%)\end{array}$ & $\begin{array}{c}\text { Total } \\
\text { aluminium } \\
(\%)\end{array}$ & $\begin{array}{c}\text { Water } \\
\text { content } \\
(\% \text { wet) }\end{array}$ & $\begin{array}{c}\text { Mean } \\
\text { size } \\
(\mu \mathrm{m})\end{array}$ & $\begin{array}{c}\text { Organic } \\
\text { carbon } \\
(\%)\end{array}$ & $\begin{array}{c}\text { Inorganic } \\
\text { carbon } \\
(\%)\end{array}$ & $\begin{array}{c}\text { Dilution } \\
\text { factor }\end{array}$ \\
\hline Site 745 & & & & & & & & \\
2.70 & 4.9 & 16.10 & 2.59 & 74.30 & 4.8 & 0.26 & 0.04 & 1.56 \\
2.20 & 4.9 & 14.50 & 3.93 & 81.50 & 4.8 & 0.34 & 0.04 & 1.59 \\
16.70 & 4.9 & 15.20 & 2.31 & 73.30 & 4.0 & 0.17 & 0.03 & 1.44 \\
45.20 & 2.2 & 6.50 & 5.49 & 60.60 & 4.5 & 0.19 & 0.01 & 1.46 \\
73.70 & 4.6 & 9.20 & 4.32 & 60.40 & 4.7 & 0.15 & 0.02 & 1.50 \\
102.20 & 4.6 & 7.00 & 4.27 & 59.70 & 4.7 & 0.11 & 0.08 & 1.53 \\
130.70 & 4.8 & 6.20 & 5.35 & 54.00 & 3.8 & 0.07 & 0.05 & 1.72 \\
141.20 & 4.8 & 11.10 & 2.43 & 67.40 & 3.4 & 0.06 & 0.10 & 1.68 \\
160.20 & 3.4 & 8.30 & 4.17 & 55.60 & 3.6 & 0.06 & 0.11 & 1.80 \\
188.70 & 3.4 & 7.10 & 5.18 & 56.80 & 4.0 & 0.21 & 0.01 & 1.90 \\
& & & & & & & & \\
Site 746 & & & & & & & & \\
176.50 & 2.4 & 5.70 & 4.48 & 60.30 & 5.3 & 0.10 & 0.01 & 1.68 \\
195.50 & 1.2 & 13.20 & 5.46 & 51.90 & 4.4 & 0.10 & 0.01 & 1.66 \\
220.00 & 0.2 & 15.30 & 4.09 & 55.50 & 4.3 & 0.13 & 0.01 & 1.75 \\
244.40 & 0.2 & 15.00 & 4.22 & 56.00 & 6.5 & 0.12 & 0.37 & 2.26 \\
\hline
\end{tabular}

Table 3. Correlation coefficients between concentration differences for the two methods.

\begin{tabular}{lccccc}
\hline & \multicolumn{5}{c}{ Difference in concentration between the two methods } \\
\cline { 2 - 6 } & Silica & Sulfate & Ammonia & Magnesium & Calcium \\
\hline $\mathrm{pE}$ & 0.0 & 0.0 & 0.45 & 0.0 & 0.0 \\
Opaline silica & 0.0 & 0.52 & 0.46 & -0.48 & 0.0 \\
Total aluminum & 0.0 & 0.0 & -0.56 & 0.0 & 0.53 \\
Water content & 0.0 & 0.60 & 0.69 & -0.72 & -0.38 \\
Mean size & 0.0 & 0.0 & 0.0 & 0.0 & 0.44 \\
Organic carbon & -0.42 & 0.0 & -0.70 & 0.67 & 0.0 \\
Inorganic carbon & 0.0 & 0.0 & 0.0 & 0.0 & 0.0 \\
Dilution factor & -0.51 & 0.0 & -0.74 & 0.81 & 0.41 \\
\hline
\end{tabular}

Note: All correlation coefficients shown are significant at $p<.001$.

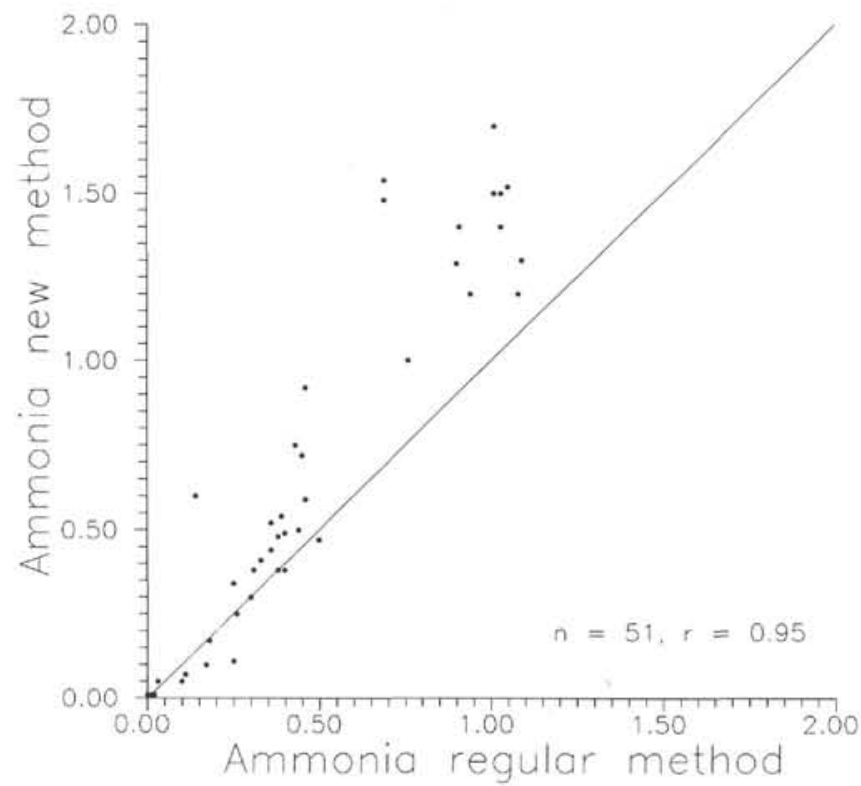

Figure 1. Comparison of ammonia concentrations (mM) between the regular and proposed interstitial-water methods.

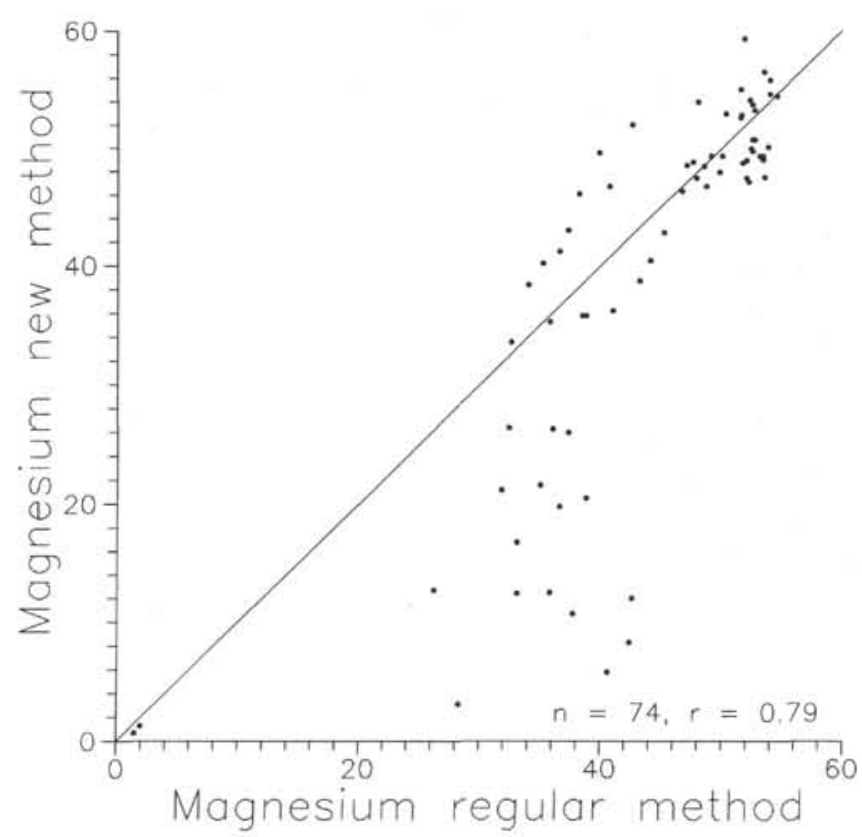

Figure 2. Comparison of magnesium concentrations (mM) between the regular and proposed interstitial-water methods.

method concentrations are an average of $1 \%$ higher than the regular method results (standard deviation of the mean $\pm 2 \%, n$ $=54$, calculated from Table 1).

\section{Calcium}

Calcium results (Fig. 3) show that the new dilution method produced low results for two samples with extremely high $\mathrm{Ca}$ from Site 737, where a major shift in equilibrium conditions caused the concentration of $\mathrm{Ca}$ to dramatically increase while $\mathrm{Mg}$ values dropped to less than $5 \%$ of normal concentrations (Barron, Larsen, et al., 1989).

In Figure 3, some of the data points at lower concentrations tend to fall below the equal-concentration line. This is the same group from Site 739 that provided exceptionally low results for 


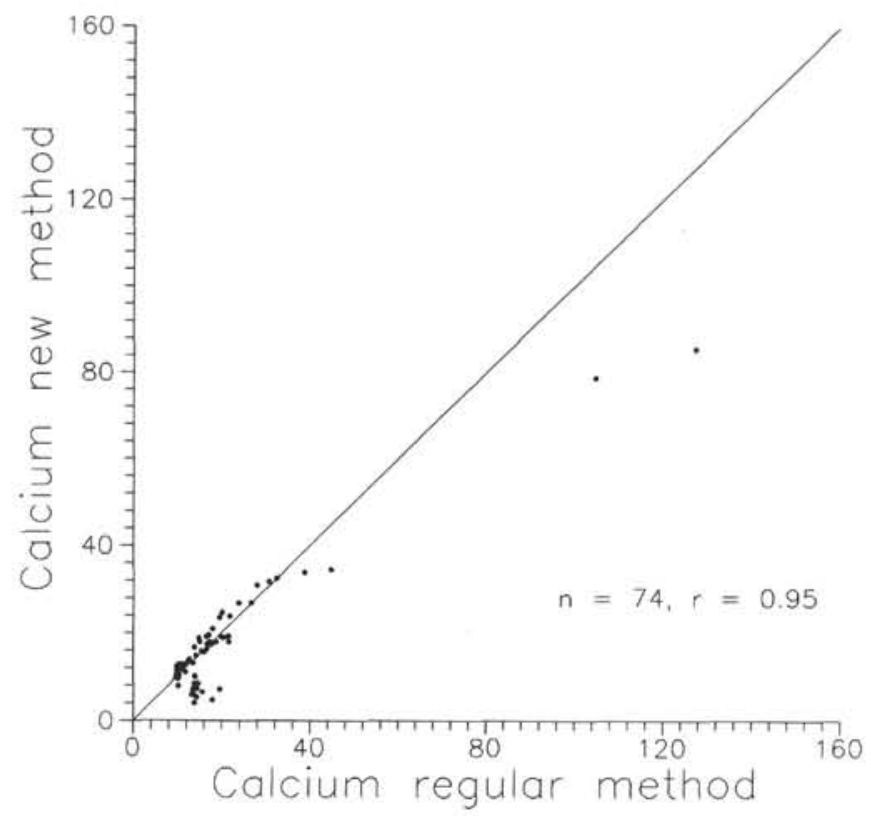

Figure 3. Comparison of calcium concentrations $(\mathrm{mM})$ between the regular and proposed interstitial-water methods.

dissolved $\mathrm{Mg}$ using the new dilution method. Differences between $\mathrm{Ca}$ concentrations determined by the two methods do not account for a majority of the variance for any of the geochemical parameters (i.e., $r<0.71$ or $r^{2}<0.5$ ). The two high Ca values for Site 737 are not part of the normal distribution, as can be determined by a t-test. As a result, when they are excluded from the correlation analyses used to produce Table 3 , the correlation between $\mathrm{Ca}$ differences and the dilution factor increases to $r=0.74$, suggesting that as dilution increases, the Ca results for the new method tend to decrease, agreeing with the earlier findings that divalent ions are lost from solution during dilution. When cases with dilution factors less than 2.5 are selected, the dilution method concentrations are an average of $4 \%$ higher than the regular method results (standard deviation of the mean $\pm 2 \%, n=54$, calculated from Table 1 ).

\section{Silica}

The new dilution method produced consistently higher results for silica relative to the routine ODP procedure (Fig. 4). The enhanced values for silica are linked to the change in ionic strength and solid/liquid ratio when deionized water is added to the sediment sample. By diluting the pore water, ion concentrations drop below equilibrium concentrations, thus allowing ions to dissolve or desorb. After correction for the dilution effect, the resulting concentrations were commonly higher than those found using the regular ODP squeezing method. From laboratory testing, I found that dissolved $\mathrm{Si}$ values would increase immediately after adding deionized water to wet sediment. Dissolution kinetics discussed by Barnard (1977) indicate that sorption/desorption reactions for silica occur within minutes of changing equilibrium conditions. When cases with dilution factors less than 2.5 are selected, the dilution method concentrations are an average of $17 \%$ higher than the regular method results (standard deviation of the mean $\pm 2 \%, n=54$, calculated from Table 1).

\section{Sulfate}

The sulfate results in Figure 5 are scattered along the equal-concentration line. Mineral particles tend to be negatively charged;

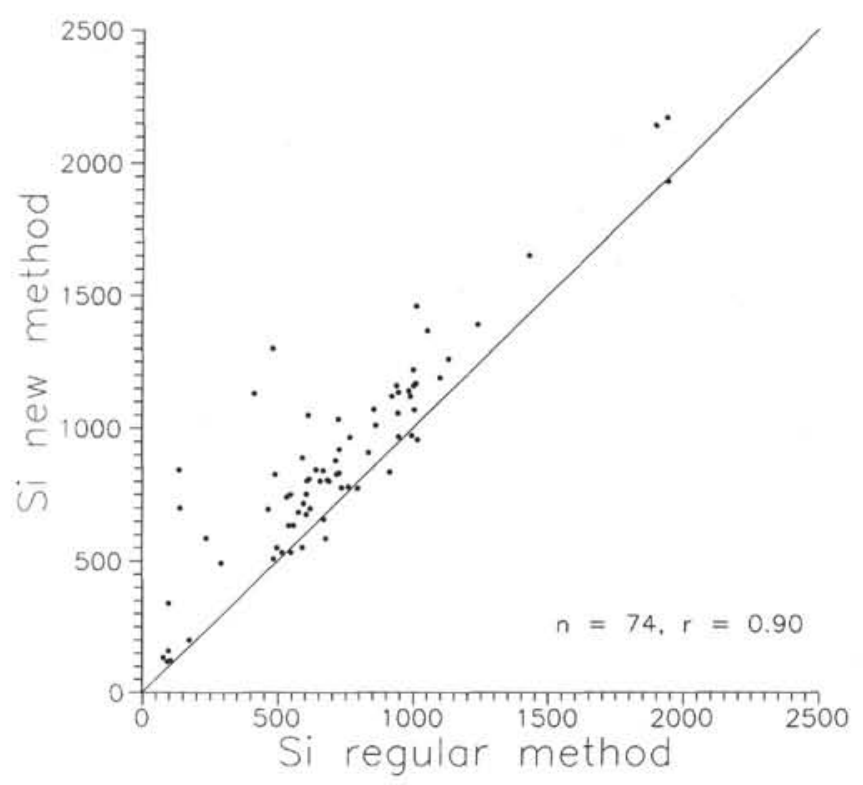

Figure 4. Comparison of silica concentrations $(\mu \mathrm{M})$ between the regular and proposed interstitial-water methods.

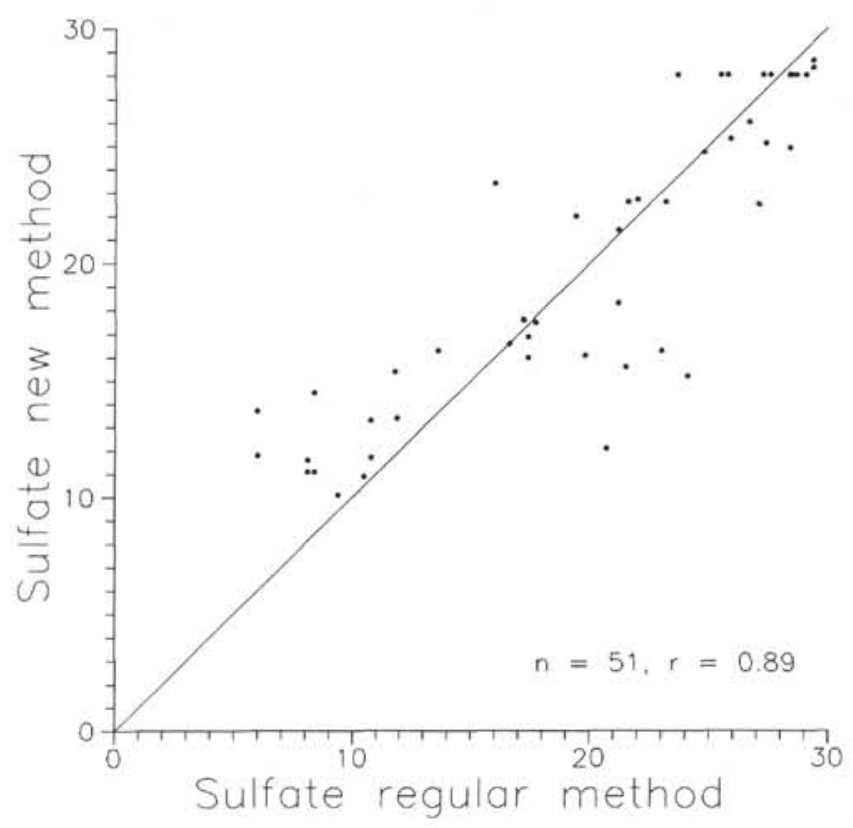

Figure 5. Comparison of sulfate concentrations $(\mathrm{mM})$ between the regular and proposed interstitial-water methods.

therefore, adsorption of anions is minimal compared to that for cations. As a result, it is not expected that sulfate concentrations will vary due to ionic exchange effects when the dilution method is used. Dilution may cause anion repulsion processes to occur (Bohn et al., 1985); however, they will be dominated by chloride ions rather than sulfate ions because of a higher concentration and charge to surface ratio for chloride.

The correlation analyses comparing the difference between sulfate methods with geochemical parameters (Table 3 ) does not produce conclusive evidence that sulfate analyses are affected by the dilution step. Some of the scatter can be attributed to problems encountered with the integrator coupled to the sulfate ana- 
lyzer, especially for the first the sites of Leg 119 . When cases with dilution factors less than $\mathbf{2 . 5}$ are selected, the dilution method concentrations are an average of $2 \%$ lower than the regular method results (standard deviation of the mean $\pm 3 \%, n=$ 36, calculated from Table 1).

\section{CONCLUSIONS}

A new pore-water sampling method provides information for a number of dissolved chemical species at higher resolution. The results are useful in a relative sense for silica, sulfate, ammonia, magnesium, and calcium. More than $80 \%$ of the variance in routine results can be accounted for by the new method if dilution factors are below 1:2.5. Even though there is some degradation in data quality, the advantages of the method are

1. sample resolution can be increased from a $30-$ to $0.3-\mathrm{m}$ spacing;

2. sample volume is reduced from 300 to $10 \mathrm{~mL}$;

3. samples with extremely low water contents can be processed;

4. pore-water recovery can be done under controlled temperature and atmospheric conditions; and

5. resampling at higher resolution in selected core sections can be carried out after routine geochemical data are examined.

The proposed method may be improved by adding only $3 \mathrm{~mL}$ (rather than $5 \mathrm{~mL}$ ) of deionized water to a $10-\mathrm{mL}$ wet sediment sample and by using a more powerful centrifuge to recover the water. Keeping the dilution factor lower will improve the data quality. The new method may be applied to other ions; however, careful testing of the methods would be required.

\section{ACKNOWLEDGMENTS}

Geological Survey of Canada Contribution no. 43789.

\section{REFERENCES}

Barnard, L. A., 1977. The kinetics and thermodynamics of silica sorption in marine sediments [M.S. thesis]. Univ. of South Florida, St. Petersburg.

Barron, J., Larsen, B., et al., 1989. Proc. ODP, Init. Repts., 119: College Station, TX (Ocean Drilling Program).

Bohn, H. L., McNeal, B. L., and O'Connor, G. A., 1985. Soil Chemistry (2nd ed.): New York (Wiley).

DeLange, G. J., Cranston, R. E., Hydes, D., and Boust, D., 1989. Problems that can be encountered during the extraction of pore-water from marine sediments. In Schuttenhelm, R.T.E., Auffret, G. A., Buckley, D. E., Cranston, R. E., Murray, C. N., Shephard, L. E., and Spijkstra, A. E. (Eds.), Geoscience Investigations of Two North Atlantic Abyssal Plains-The ESOPE International Expedition: Luxembourg (Office for Official Publications of the European Communities), EUR 12331 EN:727-770.

Loder, T. C., Lyons, W. B., Murray, S., and McGuinness, H. D., 1978. Silicate in anoxic pore-waters and oxidation effects during sampling. Nature, 273:373-374.

Sayles, F. L., and Mangelsdorf, P. C., 1977. The equilibrium of clay minerals with seawater: exchange reactions. Geochim. Cosmochim. Acta, 41:951-960.

Whitfield, M., 1969. Eh as an operational parameter in estuarine studies. Limnol. Oceanogr., 14:547-558.

Date of initial receipt: 7 December 1989

Date of acceptance: 1 August 1990

Ms 119B-173 